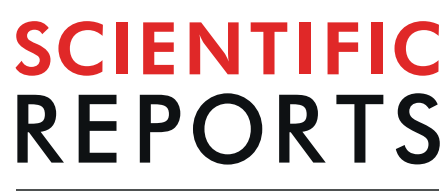

natureresearch

\title{
Enhancing the differentiation of specific genotypes in Mycobacterium tuberculosis population
}

\author{
Shima Hadifar ${ }^{1,2,3}$, Mansour Kargarpour Kamakoli $\mathbb{1}^{1,2,3}$, Abolfazl Fateh ${ }^{1,2}$, \\ Seyed Davar Siadat ${ }^{1,2}$ \& Farzam Vaziri $\mathbb{1}^{1,2^{*}}$
}

Today, significant attention is directed towards the global lineages and sublineages of $M y c o b a c t e r i u m$ tuberculosis (Mtb). NEW-1 (SIT 127) and CAS1-Delhi (SIT 26) strains are recognized as growing and circulating Mtb genotypes, especially in Asian countries. It is crucial to develop or enhance $M t b$ genotyping methods for a more accurate and simple differentiation of these families. We used 24-loci mycobacterial interspersed repetitive unit-variable number tandem repeat (MIRU-VNTR) typing for genotyping $217 \mathrm{Mtb}$ isolates. To select the optimal MIRU-VNTR loci, we calculated the Hunter-Gaston discriminatory index (HGDI), allelic diversity, and accumulation of percentage differences (APDs) between the strains among different groups of genotypes (NEW-1 and non-NEW-1; CAS1-Delhi and non-CAS). Finally, the minimum spanning tree was constructed for clustering analysis. In the NEW-1 population, loci with APD $>60 \%$ were found to have a high discriminatory power. VNTR loci with APD $>50 \%$ showed high discrimination power for the CAS population. Our findings suggest that APDs, which are valuable for the selection of VNTR loci sets, may improve the discriminatory power of MIRUVNTR typing for identification of $M t b$ genotypes in specific regions.

Today, significant attention is directed towards the global lineages of Mycobacterium tuberculosis (Mtb). Among seven recognized lineages of $M t b$, lineage 4 is the most widely dispersed, affecting humans around the world ${ }^{1}$. One of the important genotypes in lineage 4 is the NEW-1 family. In addition, NEW-1 (SIT 127) and CAS1-Delhi (SIT 26) strains have been identified as growing and circulating $M t b$ genotypes, especially in Asian countries including Iran, Afghanistan, and Pakistan ${ }^{2-7}$. In these regions, the prevalence of NEW-1 is strongly associated with multidrug resistance $(\mathrm{MDR})^{7,8}$.

The ongoing increase in the circulation of NEW-1 (SIT 127) and CAS1-Delhi strains in West of Asia seems more alarming for the NEW-1 clone population, which is prone to MDR ${ }^{9}$. These reports demonstrate the need for developing or enhancing $M t b$ genotyping methods. Overall, genotyping of $M t b$ strains plays an important role in understanding the dynamics of $M t b$ population, tuberculosis (TB) surveillance, and control programs. The 24-loci mycobacterial interspersed repetitive unit-variable number tandem repeat (MIRU-VNTR) typing is a popular and promising genotyping method for investigating the polymorphisms of $M t b$ DNA minisatellites. Compared to other PCR-based genotyping methods, such as spoligotyping, this approach is less prone to homoplasy and shows a greater discriminatory power ${ }^{10,11}$. Due to the presence of geographic polymorphisms in some VNTR loci, 24-loci VNTR sets may not be able to precisely discriminate local $M t b$ isolates. Therefore, we conducted this study to explore a VNTR loci set for discriminating NEW-1 and CAS1-Delhi (the main sub-lineages of lineages 4 and 3, respectively) from other genotypes by different approaches.

\footnotetext{
${ }^{1}$ Department of Mycobacteriology and Pulmonary Research, Pasteur Institute of Iran, Tehran, Iran. ${ }^{2}$ Microbiology Research Center (MRC), Pasteur Institute of Iran, Tehran, Iran. ${ }^{3}$ These authors contributed equally: Shima Hadifar and Mansour Kargarpour Kamakoli. *email: f_vaziri@pasteur.ac.ir
} 


\begin{tabular}{|c|c|c|c|c|c|c|c|c|c|c|}
\hline \multirow{2}{*}{$\begin{array}{l}\text { MIRU- } \\
\text { VNTR } \\
\text { locus }\end{array}$} & \multicolumn{2}{|c|}{ Total $(n=217)$} & \multicolumn{2}{|c|}{ CAS-Delhi $(\mathrm{n}=95)$} & \multicolumn{2}{|c|}{ Non-CAS $(n=122)$} & \multicolumn{2}{|c|}{ NEW-1 $(n=80)$} & \multicolumn{2}{|c|}{ Non-NEW-1 $(n=137)$} \\
\hline & $\begin{array}{l}\text { Diversity } \\
\text { Index }\end{array}$ & CI (95\%) & $\begin{array}{l}\text { Diversity } \\
\text { Index }\end{array}$ & CI (95\%) & $\begin{array}{l}\text { Diversity } \\
\text { Index }\end{array}$ & CI $(95 \%)$ & $\begin{array}{l}\text { Diversity } \\
\text { Index }\end{array}$ & CI $(95 \%)$ & $\begin{array}{l}\text { Diversity } \\
\text { Index }\end{array}$ & CI (95\%) \\
\hline 2687 & 0.009 & $(1.000-0.027)$ & 0 & $(0.000-0.000)$ & 0.016 & $(1.000-0.049)$ & 0 & $(0.000-0.000)$ & 0.015 & $(1.000-0.043)$ \\
\hline 2347 & 0.063 & $(0.017-0.109)$ & 0.021 & $(1.000-0.062)$ & 0.096 & $(0.023-0.169$ & 0.098 & $(0.007-0.189)$ & 0.043 & $(1.000-0.092)$ \\
\hline 154 & 0.072 & $(0.024-0.120)$ & 0.102 & $(0.018-0.187)$ & 0.049 & $(1.000-0.103)$ & 0.025 & $(1.000-0.073)$ & 0.099 & $(0.029-0.169)$ \\
\hline 580 & 0.137 & $(0.077-0.198)$ & 0.156 & $(0.061-0.251)$ & 0.124 & $(0.046-0.201)$ & 0.182 & $(0.075-0.290)$ & 0.111 & $(0.040-0.182)$ \\
\hline 3007 & 0.205 & $(0.133-0.277)$ & 0.196 & $(0.090-0.301)$ & 0.212 & $(0.116-0.308)$ & 0.121 & $(0.023-0.219)$ & 0.251 & $(0.157-0.345)$ \\
\hline 2531 & 0.2 & $(0.132-0.269)$ & 0.14 & $(0.046-0.235)$ & 0.244 & $(0.152-0.336)$ & 0.074 & $(1.000-0.154)$ & 0.266 & $(0.177-0.354)$ \\
\hline 3690 & 0.355 & $(0.277-0.433)$ & 0.357 & $(0.243-0.470)$ & 0.356 & $(0.250-0.461)$ & 0.271 & $(0.146-0.397)$ & 0.4 & $(0.305-0.496)$ \\
\hline 3171 & 0.445 & $(0.400-0.491)$ & 0.157 & $(0.061-0.254)$ & 0.504 & $(0.498-0.510)$ & 0.339 & $(0.234-0.444)$ & 0.112 & $(0.040-0.183)$ \\
\hline 2401 & 0.463 & $(0.395-0.532)$ & 0.488 & $(0.385-0.591)$ & 0.447 & $(0.356-0.537)$ & 0 & $(0.000-0.000)$ & 0.608 & $(0.552-0.663)$ \\
\hline 2461 & 0.529 & $(0.491-0.567)$ & 0.102 & $(0.018-0.187)$ & 0.488 & $(0.416-0.561)$ & 0.049 & $(1.000-0.116)$ & 0.189 & $(0.102-0.277)$ \\
\hline 802 & 0.481 & $(0.401-0.561)$ & 0.458 & $(0.338-0.578)$ & 0.499 & $(0.394-0.604$ & 0.508 & $(0.382-0.634)$ & 0.462 & $(0.362-0.562)$ \\
\hline 577 & 0.54 & $(0.509-0.571)$ & 0.103 & $(0.017-0.188)$ & 0.126 & $(0.045-0.208)$ & 0.097 & $(0.008-0.187)$ & 0.476 & $(0.408-0.545)$ \\
\hline 4156 & 0.595 & $(0.536-0.655)$ & 0.738 & $(0.684-0.791)$ & 0.423 & $(0.327-0.519)$ & 0.283 & $(0.164-0.402)$ & 0.706 & $(0.656-0.756)$ \\
\hline 4348 & 0.647 & $(0.605-0.688)$ & 0.678 & $(0.649-0.706)$ & 0.488 & $(0.389-0.588)$ & 0.252 & $(0.127-0.377)$ & 0.693 & $(0.673-0.713)$ \\
\hline $2163 \mathrm{~b}$ & 0.55 & $(0.474-0.626)$ & 0.362 & $(0.245-0.479)$ & 0.641 & $(0.564-0.718)$ & 0.286 & $(0.164-0.408)$ & 0.652 & $(0.577-0.727)$ \\
\hline 3192 & 0.656 & $(0.608-0.704)$ & 0.728 & $(0.694-0.761)$ & 0.536 & $(0.442-0.630)$ & 0.121 & $(0.022-0.219)$ & 0.741 & $(0.720-0.762)$ \\
\hline 2059 & 0.617 & $(0.567-0.667)$ & 0.645 & $(0.569-0.721)$ & 0.598 & $(0.532-0.663)$ & 0.611 & $(0.534-0.687)$ & 0.624 & $(0.558-0.690)$ \\
\hline 1644 & 0.679 & $(0.643-0.714)$ & 0.487 & $(0.377-0.597)$ & 0.503 & $(0.418-0.588$ & 0.38 & $(0.255-0.505)$ & 0.656 & $(0.594-0.719)$ \\
\hline 2165 & 0.705 & $(0.673-0.737)$ & 0.708 & $(0.646-0.770)$ & 0.637 & $(0.578-0.697)$ & 0.395 & $(0.272-0.518)$ & 0.653 & $(0.579-0.727)$ \\
\hline 1955 & 0.629 & $(0.589-0.669)$ & 0.598 & $(0.500-0.695)$ & 0.446 & $(0.350-0.542)$ & 0.437 & $(0.310-0.564)$ & 0.623 & $(0.568-0.678)$ \\
\hline 2996 & 0.761 & $(0.721-0.802)$ & 0.806 & $(0.768-0.845)$ & 0.632 & $(0.565-0.700)$ & 0.646 & $(0.582-0.709)$ & 0.781 & $(0.737-0.825)$ \\
\hline 424 & 0.762 & $(0.729-0.795)$ & 0.788 & $(0.745-0.830)$ & 0.617 & $(0.529-0.705)$ & 0.273 & $(0.146-0.400)$ & 0.777 & $(0.742-0.811)$ \\
\hline 960 & 0.79 & $(0.759-0.821)$ & 0.772 & $(0.732-0.811)$ & 0.502 & $(0.442-0.563)$ & 0.097 & $(0.008-0.187)$ & 0.8 & $(0.772-0.828)$ \\
\hline 4052 & 0.837 & $(0.817-0.856)$ & 0.811 & $(0.769-0.853)$ & 0.785 & $(0.741-0.830)$ & 0.684 & $(0.601-0.768)$ & 0.816 & $(0.778-0.853)$ \\
\hline
\end{tabular}

Table 1. HGDI of different MIRU-VNTR loci. *Bold value represented HGDI value difference $\geq 0.2$.

\section{Results}

According to the results of MIRU-VNTR genotyping method, 80 out of $217 \mathrm{Mtb}$ isolates were identified as NEW-1 genotype and 95 isolates as CAS1-Delhi genotype. Other isolates belonged to the Beijing genotype. Similar results were also obtained by the spoligotyping method. We aimed to identify appropriate loci with the capacity for precise classification and differentiation of genotypes; therefore, we examined the capacity of the current set of MIRU-VNTR loci.

As shown in Table 1, the discriminatory power of each locus was different between NEW-1, CAS-Delhi, and other genotypes. In the NEW-1 population, VNTRs 4052 (QUB26), 2996 (MIRU26), and 2059 (MIRU20) showed a high degree of discrimination (HGDI $>0.6$ ), whereas 16 VNTR loci were poorly discriminative (HGDI $<0.3)$. Thirteen VNTR loci showed high discrimination for non-NEW-1 genotypes, three of which, including 4052 (QUB26), 2996 (MIRU26), and 2059 (MIRU20), exhibited a high discriminatory power for the NEW-1 genotype; the remaining loci had low to moderate discriminatory power for this genotype. Eleven VNTR loci showed an HGDI difference $\geq 0.2$ between NEW- 1 and non-NEW- 1 populations. The MST plot, which was constructed based on highly discriminative VNTR loci and loci with an HGDI difference $\geq 0.2$, showed low potential to classify the genotypes into distinct branches (Fig. 1A,B).

In the CAS-Delhi population, nine VNTR loci, including 4052 (QUB26), 2996 (MIRU26), 424 (Mtub04), 960 (MIRU10), 4156 (QUB4156c), 3192 (MIRU31), 2165 (ETRA), 4348 (MIRU39), and 2059 (MIRU20), showed high discrimination, while the other nine VNTR loci exhibited low discrimination (HGDI $<0.3)$. VNTR960 (MIRU10), along with 2059 (MIRU20), 3192 (MIRU31), 4348 (MIRU39), and 4156 (QUB4156c), displayed a high discrimination power for the CAS-Delhi genotype and moderate discrimination for non-CAS genotypes. In addition, five loci showed an HGDI difference $\geq 0.2$ between CAS and non-CAS genotypes. The MST, which was constructed based on nine VNTR loci with high discrimination power and five loci with an HGDI difference $\geq 0.2$ in the CAS-Delhi population, failed to classify the genotypes into distinct branches (Fig. 2A,B).

The allelic diversity $(\mathrm{h})$ of each VNTR locus is shown in Fig. 3. A different allelic diversity was identified between the NEW-1 and CAS genotypes. The allelic profile of each VNTR locus (copy number of tandem repeats) is shown in Supplementary Figs. S1 and S2. Based on the results, we evaluated the accumulative differences in allelic diversity by calculating APDs to identify the appropriate loci. The APD of each locus related to CAS-Delhi and NEW-1 genotypes is depicted in Fig. 4. In addition, the APD of each locus related to Beijing genotype is illustrated in Supplementary Fig. S3.

The results of MST analysis, based on the APD of each locus, demonstrated that VNTR 577 (ETRC), 960 (MIRU10), 1644 (MIRU16), 2461 (ETRB), 2996 (MIRU26), and 1955 (Mtub21) could classify CAS-Delhi and non-CAS genotypes into two main populations. It was found that 93 out of 95 (97.89\%) CAS isolates were in the CAS-Delhi population branch. The APDs of the applied VNTR loci were above 50\%. MST, based on APDs 


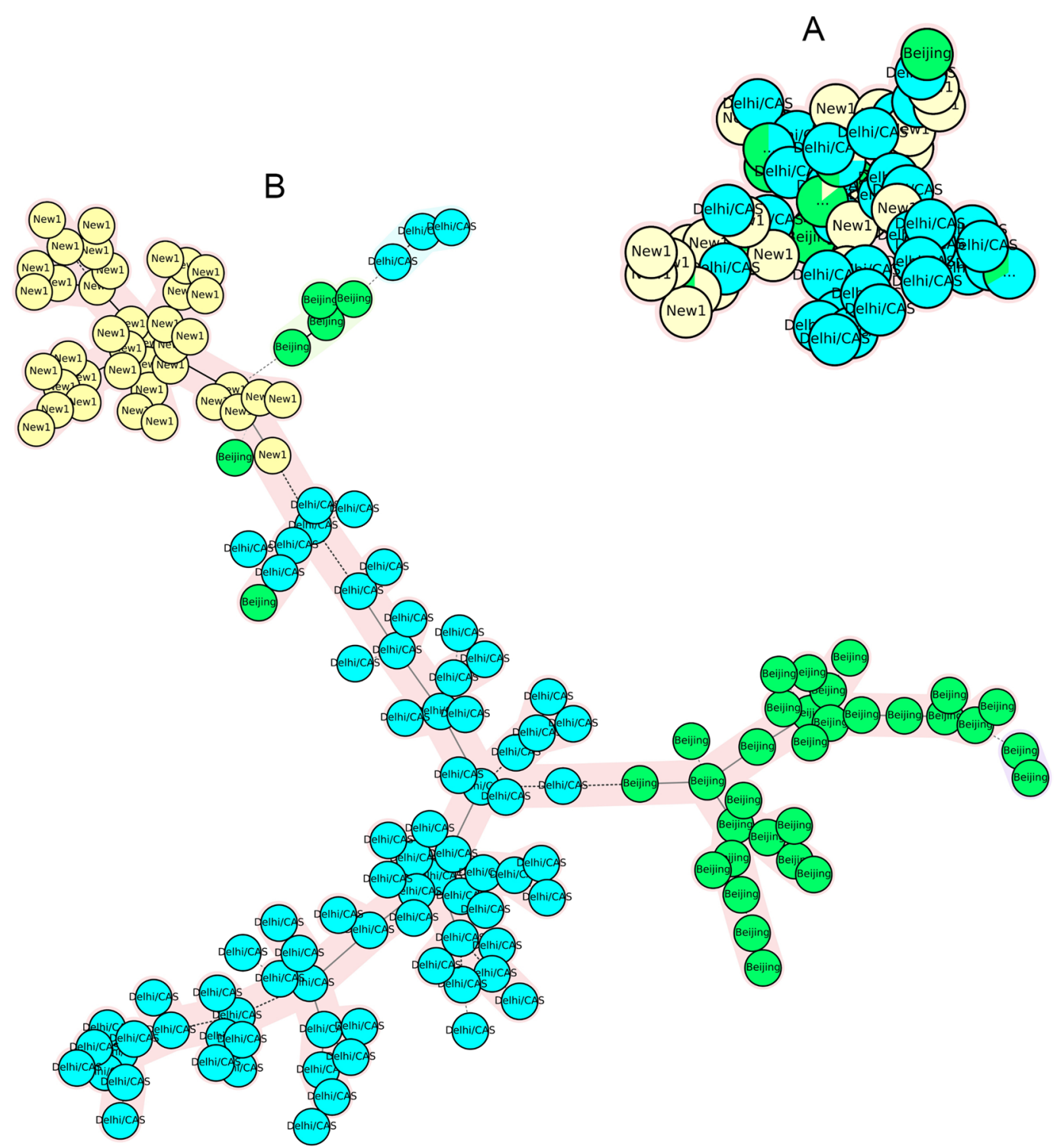

Figure 1. The MSTs of $217 \mathrm{Mtb}$ strains based on: (A) VNTR loci 4052 (QUB26), 2996 (MIRU26), and 2059 (MIRU20), HGDI > 0.6; (B) 960 (MIRU10), 424(Mtub04), 2165 (ETRA), 1644 (MIRU16), 3192 (MIRU31), 2163b (QUB11b), 4348 (MIRU39), 4156 (QUB4156), 577 (ETRC), 3171(Mtub34) and 2401 (Mtub30). NEW-1 and non-NEW-1 strains are shown in different colors.

ranging from $8 \%$ to $47 \%$, was also constructed (Fig. 5A,B). The results showed that the VNTR loci set had a low capacity for classification of CAS-Delhi and non-CAS genotypes into distinct branches, while it showed promising capacity for clustering the NEW-1 genotype. This potential is attributed to the presence of five VNTR loci with high APD values for clustering the NEW-1 population. We found loci with APDs $>50 \%$ and high discrimination power for the CAS population. In NEW-1 and non-NEW-1 genotypes, VNTR loci, including 2461 (ETRB), 960 (MIRU10), 424 (Mtub04), 3171 (Mtub34), and 577 (ETRC), could classify these isolates into two distinct groups. Out of 80 NEW-1 strains, 79 (98.75\%) were found in a distinct branch. In this classification, APDs of VNTR loci were above $70 \%$. The same result was obtained using eight loci with APDs ranging from $60 \%$ to $95 \%$. The MST, based on the other VNTR sets with an ascending order in terms of APD, showed different results and low capacity for clustering different genotypes in a specific branch (Fig. 6A-C). In the NEW-1 population, loci with $\mathrm{APD}>60 \%$ were found to have a high discriminatory power.

\section{Discussion}

The allelic diversity and discriminatory power of VNTR loci in 15/24-loci MIRU-VNTR typing have been evaluated in different studies. The results of these studies showed that the discriminatory power may be affected by the geographical distribution of isolates and strain polymorphisms in specific regions. Some studies have suggested several optimized VNTR sets, mainly VNTRs with high discriminatory powers, for improving the standard $M t b$ typing method and identifying certain genotypes ${ }^{3,12-16}$. In our study, the prominent genotypes belonged 


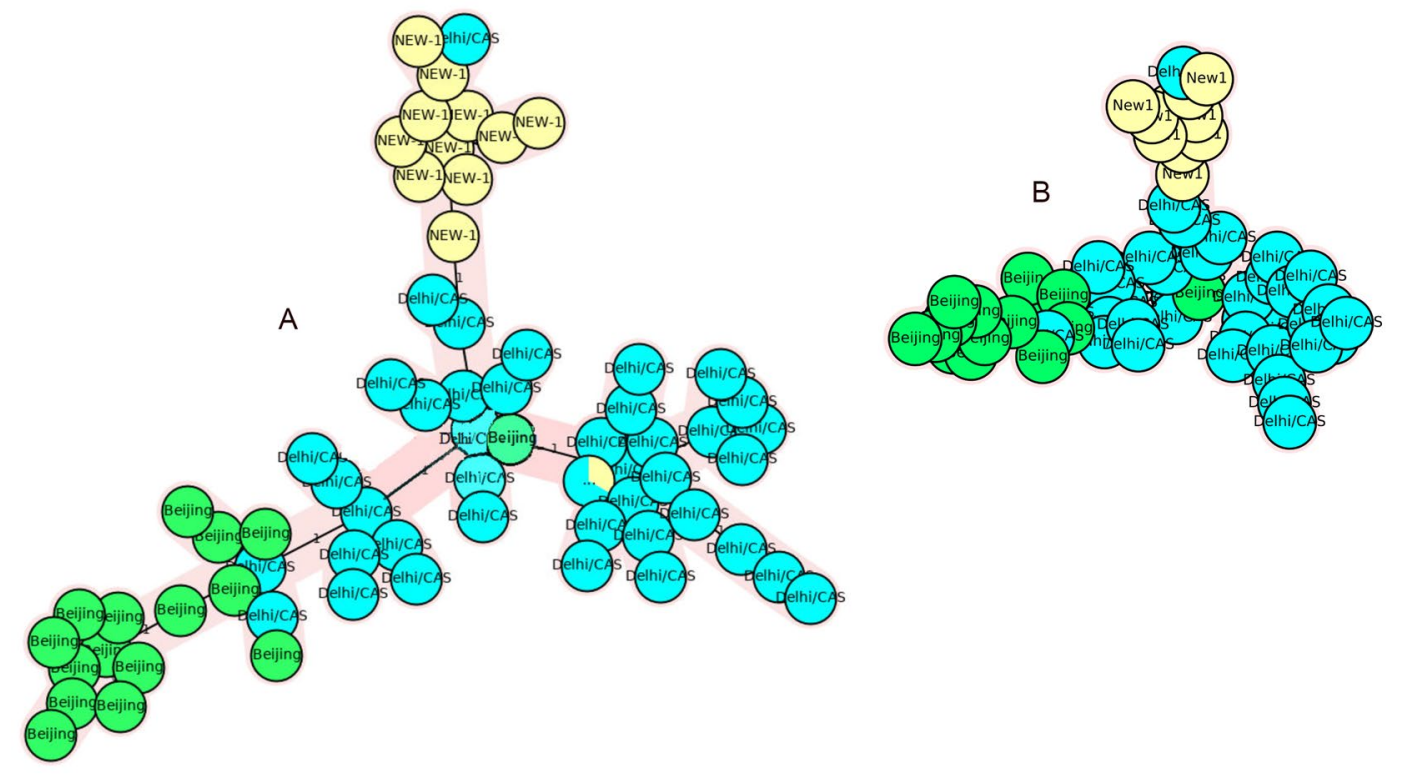

Figure 2. The MSTs of 217 Mtb strains based on: (A) VNTR loci 4052 (QUB26), 2996 (MIRU26), 424 (Mtub04), 960 (MIRU10), 4156 (QUB4156), 3192 (MIRU31), 2165 (ETRA), 4348 (MIRU39), and 2059 (MIRU20), HGDI > 0.6; (B) VNTR loci 960 (MIRU10), 4156 (QUB4156), 2163b(QUB11b), 2461 (ETRB), and 3171 (Mtub34). CAS1-Delhi and non-CAS strains are shown in different colors.

to CAS1-Delhi (SIT 26) and NEW-1 (SIT 127) families. The dispersal of NEW-1/SIT127 shows that the family initially emerged in Iran and then spread to other geographical regions ${ }^{9,17}$.

A set of eight optimized VNTR loci, including 4052 (QUB26), 2163B (QUB11b), 802 (MIRU40), 4165 (QUB4156), 2165 (ETRA), 960 (MIRU10), 1644 (MIRU16), and 2996 (MIRU26), with high HGDI values, was identified by Asante-Poku et al. for identification of lineages 4 and 5 (M. africanum West Africa-1) in Ghana ${ }^{18}$. In addition, Ali et al. reported that MIRU loci 10, 16,26, 27, 31, 39, and 40 showed high discriminatory power for separating predominant CAS1 strains in Pakistan ${ }^{15}$. In a study which conducted in Madagascar, VNTR loci 960 (MIRU10), 4156 (Qub4156), 1644 (MIRU16), 802 (MIRU40), 577 (ETRC), 3690 (Mtub39), 4052 (Qub26), 2163b (Qub11b), 3192(MIRU31) and 424 (Mtub04) have been reported as a set of ten optimized VNTR loci for discrimination of CAS sub-lineage based on HGDI value. In addition, QUB26, Mtub39, QUB4156, ETR-A, ETR-D, ETR-E, QUB11b, Mtub21, and MIRU40; QUB11b, MIRU10, QUB4156, and MIRU40; QUB26, MIRU40, and QUB11b; and Mtub39, MIRU16, QUB11b, MIRU26, ETR-C, ETR-A Qub26, MIRU40, Mtub21, MIRU10, Mtub04, QUB4156 and Mtub30 have been proposed as the set of MIRU-VNTR loci combination for discrimination of EIA, Haarlem, LAM and T genotypes, resectively ${ }^{19}$. In this study, we investigated the discriminatory power of different numbers and sets of VNTR loci to identify a combination of loci for discriminating CAS and NEW-1 from other strains. Besides 4052 (QUB26) and 2996 (MIRU26), Seven VNTR loci, namely 424 (Mtub04), 960 (MIRU10), 4156 (QUB4156), 3192 (MIRU31), 2165 (ETRA), 4348 (MIRU39), and 2059 (MIRU20), showed high discriminatory power in an ascending order for the separation of CAS-Delhi. On the other hand, the MST analysis based on this set showed low capacity for separation of CAS population in a distinct branch. A similar result was found in the MST analysis of the NEW-1 population.

For improving the loci set selection, we examined APDs, i.e., the relative difference between strains with the same repeat number at a specific VNTR locus, between CAS and non-CAS, as well as NEW-1 and non-NEW-1 strains. We found that a six-loci combination with APDs ranging from $55.53 \%$ to $96 \%$ had significant power to discriminate the CAS family, compared to another set with APDs of less than 50\%. In addition, we found two VNTR loci, namely 4052 (QUB26) and 2996 (MIRU26), with extremely high discrimination values (HGDI >0.8) and high allelic diversity (h) for CAS classification. The VNTR locus 2996 (MIRU26) showed an APD above 55\%. Considering their variations, these two loci would be excluded. Nevertheless, the locus APD showed that this locus might contribute to the definition of an effective locus set for determination of CAS strains.

In NEW-1 strains, an effective classification was obtained using a five-loci combination with APDs above $70 \%$ and an eight-loci combination with APDs above 60\%. In a previous study, the ETR-B (VNTR 2461) locus was proposed as a preliminary marker for identifying the NEW-1 genotypes ${ }^{17}$. The present results showed that this VNTR locus had low allelic diversity and discriminatory power, while it showed the highest APD (95.31\%) and was a member of VNTR locus set, which efficiently discriminated NEW-1 (SIT127) strains from non-NEW-1 strains. Moreover, these combination set in both genotypic groups have not been reported for discrimination of other M.tb sub-lineages. In a similar study, Pan et al. reported that use of APDs facilitates the identification of effective VNTR loci to discriminate the Beijing genotype as the most common family in China ${ }^{20}$. In the current study, we also used APD for the identification of effective VNTR loci to discriminate of Beijing strains from non-Beijing strains and we found that MIRU10, QUB11b, Mtub30, ETRA, ETRC, and MIRU31 had the APD 

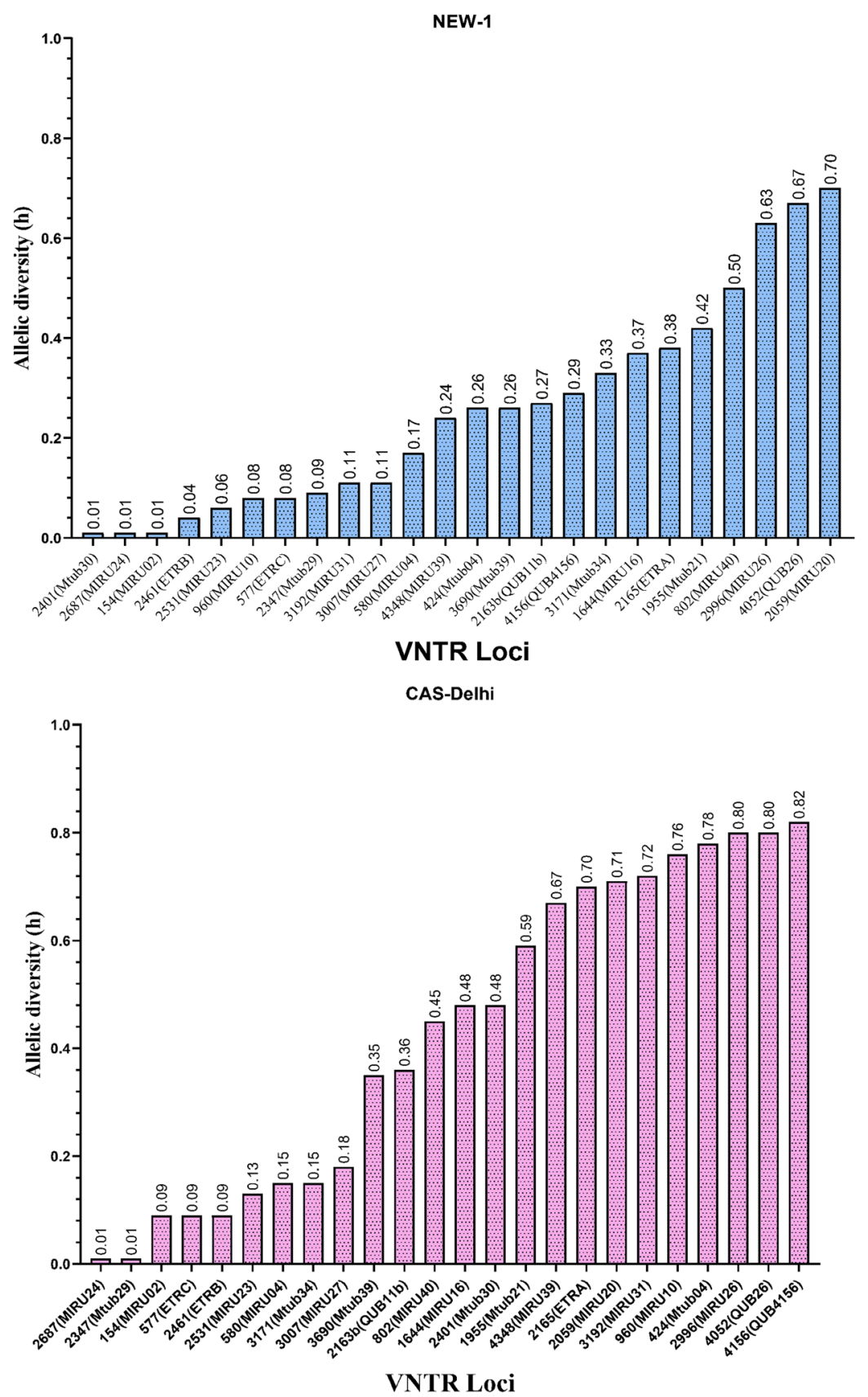

Figure 3. The allelic diversity of each MIRU-VNTR locus.

value above 50\% while Pan's study ${ }^{20}$ showed QUB18, MIRU31, Mtub30, Mtub21, MIRU10, and MIRU39 were effective VNTR loci set for discrimination of the Beijing genotypes. The specific geographical setting, the particular circulating sub-lineage and the different sample size may explain this difference in the proposed VNTR loci combination.

Our findings suggest that APDs, which are valuable in the selection of VNTR loci sets, may improve the discriminatory power of MIRU-VNTR typing for identification of Mtb genotypes (NEW-1 and CAS1-Delhi) in specific regions. However, further investigations with combined SNPs and VNTR typing schemes can improve the identification and classification of these monomorphic bacterial pathogens.

\section{Methods}

Isolate collection. We randomly collected $217 \mathrm{Mtb}$ isolates from individuals with definite pulmonary TB infection from January 2015 to January 2017. The sample included 84 isolates, which had been identified in our previous study ${ }^{2}$. The Ethics Committee of Pasteur Institute of Iran approved this study. All experiments were performed in accordance with guidelines approved by Pasteur Institute International networks. Ethical reviews and informed consent approval were granted by the Ethical Committee of the Pasteur Institute of Iran. Informed 

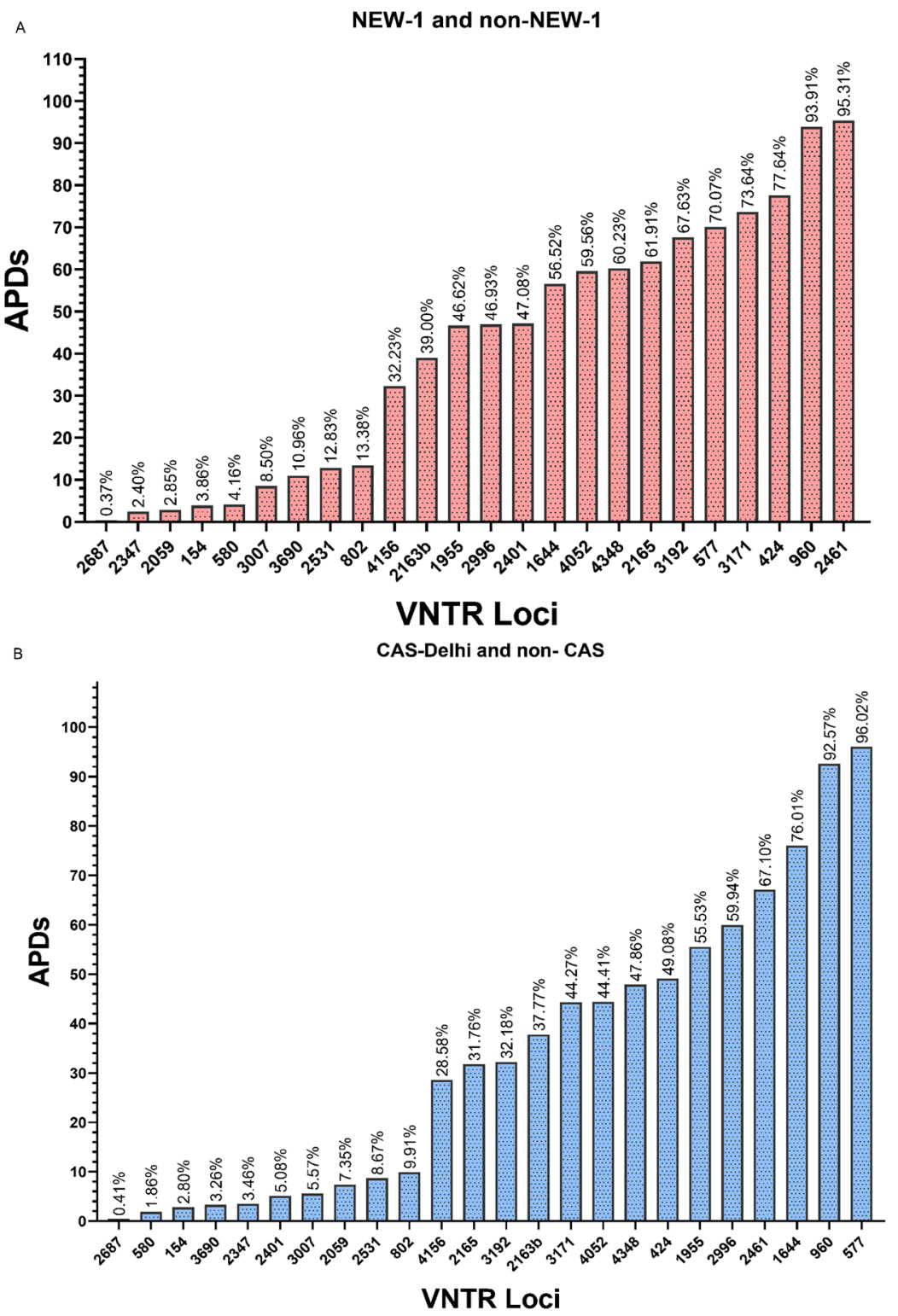

Figure 4. The APDs of 24 VNTR loci between (A) NEW-1 and non-NEW-1 strains; and (B) CAS-Delhi and non-CAS strains.

consent was obtained from all patients in this study. The results of this research did not influence TB patients' treatment.

Twenty-four-loci MIRU-VNTR typing. The genomic DNA of all inactivated $M t b$ isolates was extracted using a PROBA-NK DNA extraction kit (DNA Technology Company, Moscow, Russia) according to the manufacturer's instructions and analyzed, based on the 24-loci MIRU-VNTR genotyping method described by Supply et al. ${ }^{21}$. We calculated the allelic diversity $(\mathrm{h})^{22}$ and discriminatory power of each VNTR locus, using the Hunter-Gaston discriminatory index (HGDI), as a measure of variation in the number of repeats ${ }^{23}$ for all $M t b$ isolates. We considered $h>0.6$ as highly discriminative, $0.3 \leq h \leq 0.6$ as moderately discriminative, and $h<0.3$ as poorly discriminative ${ }^{24}$. The HGDI varies between zero and one and represents the discriminatory power for VNTR loci in combination. Besides, accumulation of percentage differences (APDs) was calculated in each VNTR locus, as described previously ${ }^{20}$.

Spoligotyping. Spoligotyping was performed using a Spoligotyping commercial kit (Mapmygenome Genomics Company, India), following the protocol described by Kamerbeek et al. ${ }^{25}$.

Data analysis and statistical analysis. The MIRU-VNTR results were analyzed in MIRU-VNTRplus (http://www.miru vntrplus.org/MIRU/index.faces) ${ }^{26}$, TBminer databases (http://infodemo.lirmm.fr/tbminer/ about.php) ${ }^{27}$ and StackTB (https://stacktb.cs.sfu.ca/) ${ }^{28}$. Based on each VNTR loci set, a Minimum Spanning Tree (MST) was constructed using MIRU-VNTRplus database to determine genetic variations and classify the isolates. 

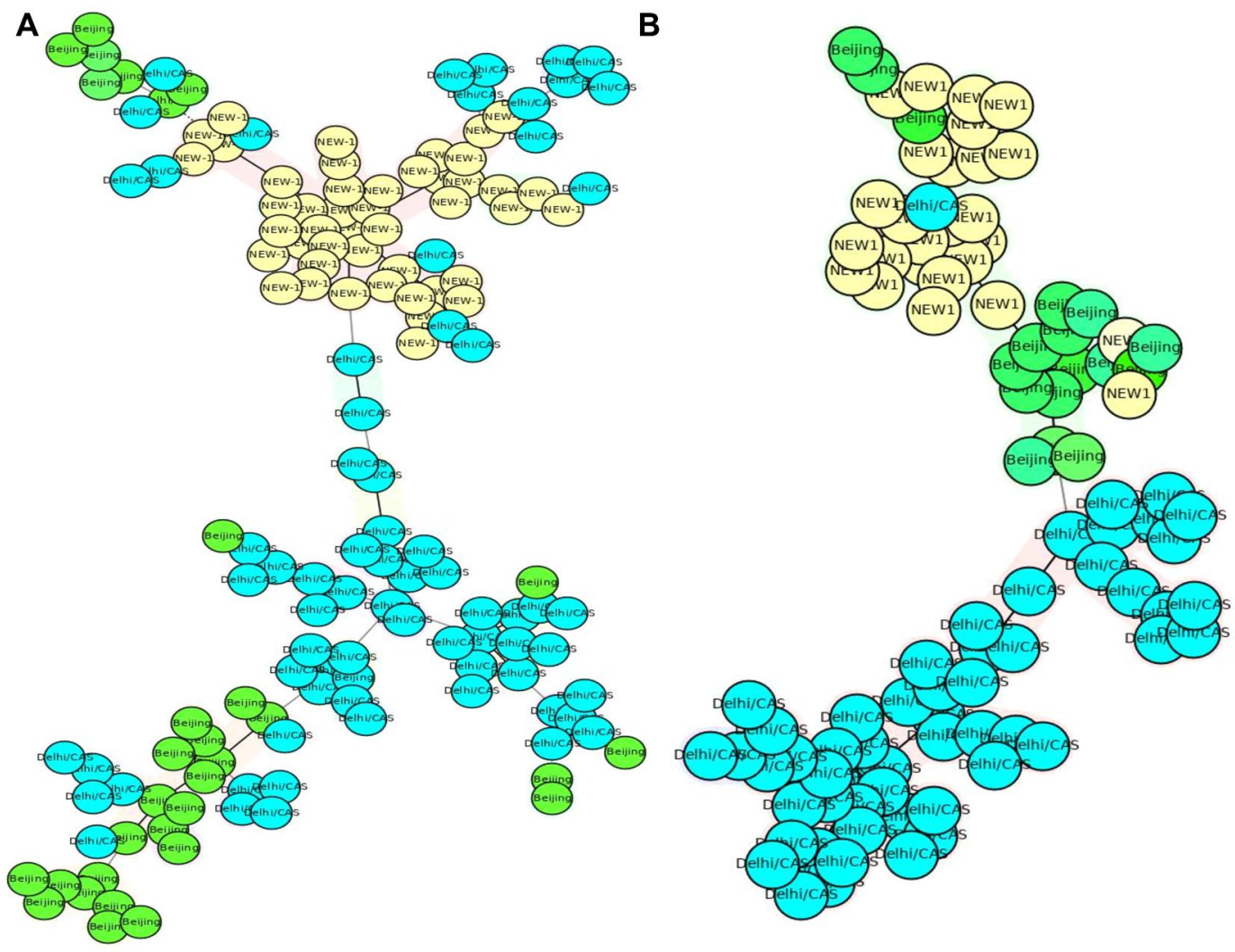

Figure 5. The MSTs of 217 Mtb strains based on: (A) VNTR 577 (ETRC), 960 (MIRU10), 1644 (MIRU16), 2461 (ETRB), 2996 (MIRU26), and 1955 (Mtub21); (B) VNTR 2531 (MIRU23), 802 (MIRU40), 4156 (QUB4156), 2165 (ETRA), 3192 (MIRU31), 2163b (QUB11b), 3171 (Mtub34), 4052 (QUB26), and 4348 (MIRU39). CAS1Delhi and non-CAS strains are shown in different colors.
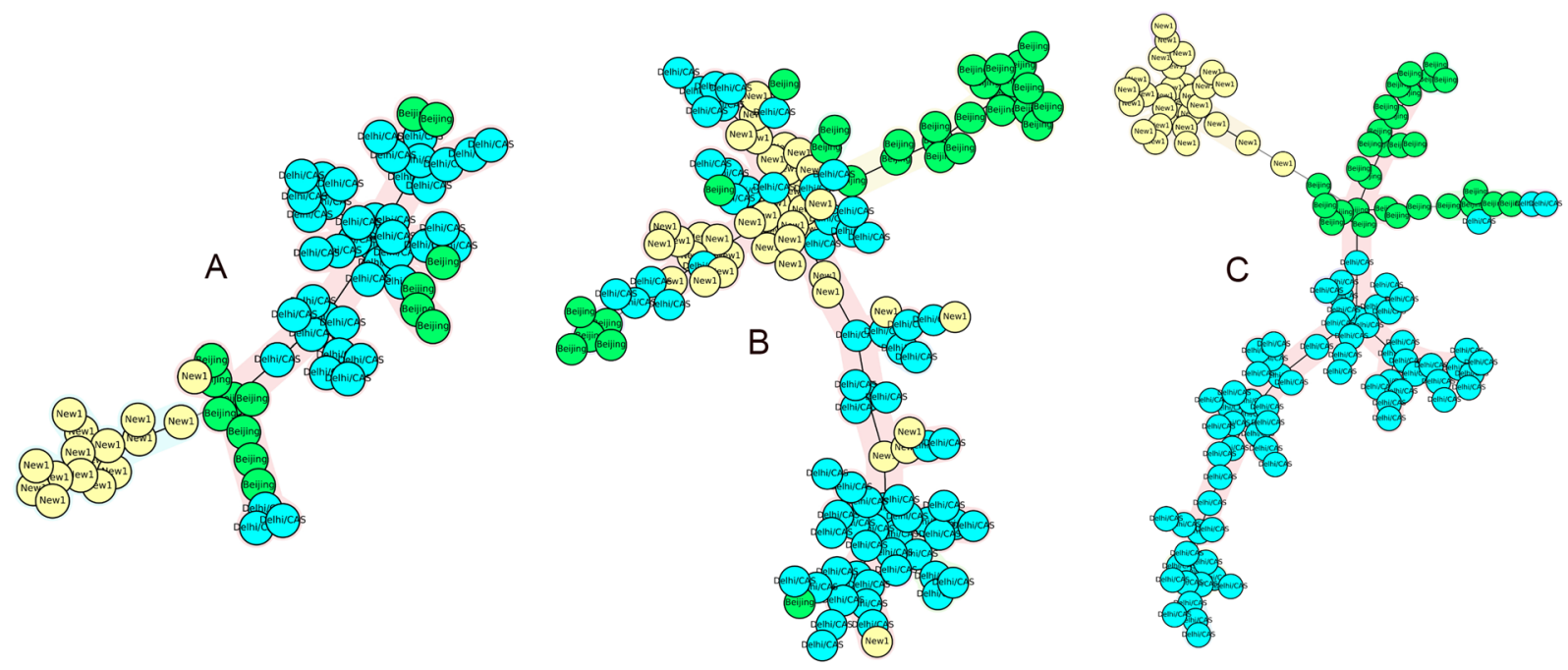

Figure 6. The MSTs of $217 \mathrm{Mtb}$ strains based on: (A)VNTR loci 2461 (ETRB), 960 (MIRU10), 424 (Mtub04), 3171 (Mtub34), and 577 (ETRC); (B) VNTR 3007 (MIRU27), 3690 (Mtub39), 2531 (MIRU23), 802 (MIRU40), 4156 (QUB4156), 2163b (QUB11b), 1955 (Mtub21), 2996 (MIRU26), and 2401 (Mtub30); (C) VNTR loci 2461 (ETRB), 960 (MIRU10), 424 (Mtub04), 3171 (Mtub34), 577 (ETRC), 3192 (MIRU31), 2165 (ETRA), and 4348 (MIRU39). NEW-1 and non-NEW-1 strains are shown in different colors.

SITVIT-WEB (http://www.pasteur-guadeloupe.fr:8081/SITVIT_ONLINE/index.jsp) ${ }^{29}$ and TBminer databases were also used to analyze the spoligotyping data.

Index of Diversity and confidence intervals were calculated by using the Web tools: (http://www.comparingpartitions.info/index.php) and (http://www.hpa-bioinfotools.org.uk/cgi-bin/DICI/DICI.pl). 
Ethics approval and consent to participate. Ethical reviews and informed written consent were granted by the Ethical Committee of the Pasteur Institute of Iran (Under the Ethics code of 927).

\section{Data availability}

The datasets used and/or analyzed during the current study could become available through the corresponding author on reasonable request.

Received: 7 June 2019; Accepted: 14 November 2019;

Published online: 29 November 2019

\section{References}

1. Brynildsrud, O. B. et al. Global expansion of Mycobacterium tuberculosis lineage 4 shaped by colonial migration and local adaptation. Science Advances 4, eaat5869 (2018).

2. Hadifar, S. et al. Genetic diversity of Mycobacterium tuberculosis isolates causing pulmonary and extrapulmonary tuberculosis in the capital of Iran. Molecular phylogenetics and evolution 132, 46-52 (2019).

3. Mansoori, N., Yaseri, M., Vaziri, F. \& Douraghi, M. Genetic diversity of Mycobacterium tuberculosis complex isolates circulating in an area with high tuberculosis incidence: Using 24-locus MIRU-VNTR method. Tuberculosis (Edinburgh, Scotland) 112, 89-97, https://doi.org/10.1016/j.tube.2018.08.003 (2018).

4. Haeili, M. et al. Spoligotyping and drug resistance patterns of Mycobacterium tuberculosis isolates from five provinces of Iran. Microbiologyopen 2, 988-996 (2013).

5. Merza, M. A., Farnia, P., Salih, A. M., Masjedi, M. R. \& Velayati, A. A. The most predominant spoligopatterns of Mycobacterium tuberculosis isolates among Iranian, Afghan-immigrant, Pakistani and Turkish tuberculosis patients: a comparative analysis. Chemotherapy 56, 248-257 (2010).

6. Hasan, Z. et al. Spoligotyping of Mycobacterium tuberculosis isolates from Pakistan reveals predominance of Central Asian Strain 1 and Beijing isolates. Journal of clinical microbiology 44, 1763-1768 (2006).

7. Ayaz, A. et al. Characterizing Mycobacterium tuberculosis isolates from Karachi, Pakistan: drug resistance and genotypes. International Journal of Infectious Diseases 16, (e303-e309 (2012).

8. Khanipour, S. et al. Haarlem 3 is the predominant genotype family in multidrug-resistant and extensively drug-resistant Mycobacterium tuberculosis in the capital of Iran: A 5-year survey. Journal of Global Antimicrobial Resistance 5, 7-10, https://doi. org/10.1016/j.jgar.2016.01.007 (2016).

9. Mokrousov, I. Emerging resistant clone of Mycobacterium tuberculosis in west Asia. The Lancet Infectious Diseases 16, 1326-1327 (2016).

10. De Beer, J., Ködmön, C., Van der Werf, M., Van Ingen, J. \& Van Soolingen, D. The ECDC MDR-TB molecular surveillance project participants. Molecular surveillance of multi-and extensively drug-resistant tuberculosis transmission in the European Union from 2003 to 2011. Euro Surveill 19, 20742 (2014).

11. De Beer, J. L. et al. First worldwide proficiency study on variable-number tandem-repeat typing of Mycobacterium tuberculosis complex strains. Journal of clinical microbiology 50, 662-669 (2012).

12. Zhao, Y. et al. The population structure of drug-resistant Mycobacterium tuberculosis clinical isolates from Sichuan in China. Infection, Genetics and Evolution 12, 718-724 (2012).

13. Luo, T. et al. Development of a hierarchical variable-number tandem repeat typing scheme for Mycobacterium tuberculosis in China. PloS one 9 , e89726 (2014).

14. Iwamoto, T. et al. Hypervariable loci that enhance the discriminatory ability of newly proposed 15 -loci and 24 -loci variable-number tandem repeat typing method on Mycobacterium tuberculosis strains predominated by the Beijing family. FEMS microbiology letters 270, 67-74 (2007).

15. Ali, A. et al. Characterization of Mycobacterium tuberculosis Central Asian Strain1 using mycobacterial interspersed repetitive unit genotyping. BMC microbiology 7, 76 (2007).

16. Shamputa, I. C. et al. Genetic diversity of Mycobacterium tuberculosis isolates from a tertiary care tuberculosis hospital in South Korea. Journal of clinical microbiology 48, 387-394 (2010).

17. Mokrousov, I. et al. Emerging peak on the phylogeographic landscape of Mycobacterium tuberculosis in West Asia: definitely smoke, likely fire. Molecular phylogenetics and evolution 116, 202-212 (2017).

18. Asante-Poku, A. et al. Evaluation of customised lineage-specific sets of MIRU-VNTR loci for genotyping Mycobacterium tuberculosis complex isolates in Ghana. PloS one 9 , e92675 (2014).

19. Rasoahanitralisoa, R. et al. Evaluation of spoligotyping, SNPs and customised MIRU-VNTR combination for genotyping Mycobacterium tuberculosis clinical isolates in Madagascar. PloS one 12, e0186088 (2017).

20. Pan, X.-L. et al. A quantitative and efficient approach to select MIRU-VNTR loci based on accumulation of the percentage differences of strains for discriminating divergent Mycobacterium tuberculosis sublineages. Emerging microbes \& infections 6, 1-8 (2017).

21. Supply, P. et al. Proposal for standardization of optimized mycobacterial interspersed repetitive unit-variable-number tandem repeat typing of Mycobacterium tuberculosis. Journal of clinical microbiology 44, 4498-4510 (2006).

22. Selander, R. K. et al. Methods of multilocus enzyme electrophoresis for bacterial population genetics and systematics. Applied and environmental microbiology 51, 873 (1986).

23. Hunter, P. R. \& Gaston, M. A. Numerical index of the discriminatory ability of typing systems: an application of Simpson's index of diversity. Journal of clinical microbiology 26, 2465-2466 (1988).

24. Sola, C. et al. Genotyping of the Mycobacterium tuberculosis complex using MIRUs: association with VNTR and spoligotyping for molecular epidemiology and evolutionary genetics. Infection, Genetics and Evolution 3, 125-133 (2003).

25. Kamerbeek, J. et al. Simultaneous detection and strain differentiation of Mycobacterium tuberculosis for diagnosis and epidemiology. Journal of clinical microbiology 35, 907-914 (1997).

26. Weniger, T., Krawczyk, J., Supply, P., Niemann, S. \& Harmsen, D. MIRU-VNTRplus: a web tool for polyphasic genotyping of Mycobacterium tuberculosis complex bacteria. Nucleic acids research 38, W326-331, https://doi.org/10.1093/nar/gkq351 (2010).

27. Azé, J. et al. Genomics and Machine Learning for Taxonomy Consensus: The Mycobacterium tuberculosis Complex Paradigm. PLOS ONE 10, e0130912, https://doi.org/10.1371/journal.pone.0130912 (2015).

28. Thain, N. et al. Towards better prediction of Mycobacterium tuberculosis lineages from MIRU-VNTR data. Infection, Genetics and Evolution 72, 59-66, https://doi.org/10.1016/j.meegid.2018.06.029 (2019).

29. Demay, C. et al. SITVITWEB-a publicly available international multimarker database for studying Mycobacterium tuberculosis genetic diversity and molecular epidemiology. Infection, genetics and evolution: journal of molecular epidemiology and evolutionary genetics in infectious diseases 12, 755-766, https://doi.org/10.1016/j.meegid.2012.02.004 (2012). 


\section{Acknowledgements}

We would like to thank all the personnel of Mycobacteriology and Pulmonary Research Department of Pasteur Institute of Iran for their assistance. This work was supported by a grant [Number 927] from Pasteur Institute of Iran.

\section{Author contributions}

S.H. and F.V. wrote the manuscript. F.V. supervised the project. F.V., S.D.S. and A.F. designed the project. S.H. and M.K.K. performed laboratory work. S.H. and M.K.K. performed the data analysis. All authors have read and approved the final manuscript.

\section{Competing interests}

The authors declare no competing interests.

\section{Additional information}

Supplementary information is available for this paper at https://doi.org/10.1038/s41598-019-54393-7.

Correspondence and requests for materials should be addressed to F.V.

Reprints and permissions information is available at www.nature.com/reprints.

Publisher's note Springer Nature remains neutral with regard to jurisdictional claims in published maps and institutional affiliations.

(c) (i) Open Access This article is licensed under a Creative Commons Attribution 4.0 International License, which permits use, sharing, adaptation, distribution and reproduction in any medium or format, as long as you give appropriate credit to the original author(s) and the source, provide a link to the Creative Commons license, and indicate if changes were made. The images or other third party material in this article are included in the article's Creative Commons license, unless indicated otherwise in a credit line to the material. If material is not included in the article's Creative Commons license and your intended use is not permitted by statutory regulation or exceeds the permitted use, you will need to obtain permission directly from the copyright holder. To view a copy of this license, visit http://creativecommons.org/licenses/by/4.0/.

(c) The Author(s) 2019 